PROCEEDINGS OF THE

AMERICAN MATHEMATICAL SOCIETY

Volume 130, Number 1, Pages 129-138

S 0002-9939(01)06023-3

Article electronically published on May 25, 2001

\title{
STABILITY OF DISJOINTNESS PRESERVING MAPPINGS
}

\author{
GREGOR DOLINAR
}

(Communicated by Dale Alspach)

\begin{abstract}
Let $X$ and $Y$ be compact Hausdorff spaces and let $\varepsilon \geq 0$. A linear mapping $\Phi: \mathcal{C}(X) \rightarrow \mathcal{C}(Y)$ is called $\varepsilon$-disjointness preserving if $f g=0$ implies that $\|\Phi(f) \Phi(g)\| \leq \varepsilon\|f\|\|g\|$. If $\Phi: \mathcal{C}(X) \rightarrow \mathcal{C}(Y)$ is a continuous or surjective $\varepsilon$-disjointness preserving linear mapping, we prove that there exists a disjointness preserving linear mapping $\Psi: \mathcal{C}(X) \rightarrow \mathcal{C}(Y)$ satisfying $\|\Phi(f)-\Psi(f)\| \leq 20 \sqrt{\varepsilon}\|f\|$. We also prove that every unbounded $\varepsilon$-disjointness preserving linear functional on $\mathcal{C}(X)$ is disjointness preserving.
\end{abstract}

\section{Introduction And StATEMENT OF the RESUlts}

In the last few years stability problems for homomorphisms and isometries have attracted the attention of many mathematicians. Among the deepest achievements in this area are Johnson's results on linear maps between Banach algebras that are approximately multiplicative [4], [5] (see also [3]). Specializing to the case of function algebras, we treat the more general problem of stability of linear mappings that preserve only zero products. Following the terminology from Banach lattices we say that a linear mapping $\Phi$ between $\mathcal{C}(X)$ and $\mathcal{C}(Y)$ is disjointness preserving if $f g=0$ implies that $\Phi(f) \Phi(g)=0$, where $f, g \in \mathcal{C}(X)$. Here and throughout the paper $\mathcal{C}(X)$ and $\mathcal{C}(Y)$ are algebras of $\mathbb{F}$-valued continuous functions defined on compact Hausdorff spaces $X$ and $Y$, respectively, and $\mathbb{F}$ is either the field of complex numbers $\mathbb{C}$ or the field of real numbers $\mathbb{R}$. Suppose a mapping $\Phi$ satisfies the disjointness preserving property only approximately. Our purpose is to determine whether there exists a disjointness preserving mapping close to it.

Let $\varepsilon \geq 0$. A linear mapping $\Phi: \mathcal{C}(X) \rightarrow \mathcal{C}(Y)$ is called $\varepsilon$-disjointness preserving if $\|\Phi(f) \Phi(g)\| \leq \varepsilon\|f\|\|g\|$ for any $f, g \in \mathcal{C}(X)$ satisfying $f g=0$.

In our first theorem we show that the disjointness preserving property is stable for continuous linear functionals on $\mathcal{C}(X)$.

Theorem 1. Let $X$ be a compact Hausdorff space and $\varepsilon \geq 0$. Then for every continuous $\varepsilon$-disjointness preserving linear functional $\Phi$ on $\mathcal{C}(X)$ there exists a continuous disjointness preserving linear functional $\Psi$ on $\mathcal{C}(X)$ satisfying

$$
|\Phi(f)-\Psi(f)| \leq 3 \sqrt{\varepsilon}\|f\| \quad \text { for all } f \in \mathcal{C}(X) .
$$

Received by the editors November 19, 1999 and, in revised form, June 9, 2000.

2000 Mathematics Subject Classification. Primary 46J10; Secondary 46E05.

Key words and phrases. $\varepsilon$-disjointness preserving mapping, stability of disjointness preserving mappings. 
If $\Phi$ is a linear mapping from $\mathcal{C}(X)$ to $\mathcal{C}(Y)$, then for every $y \in Y$ we define a linear functional $\Phi_{y}=\delta_{y} \circ \Phi$, where $\delta_{y}$ is evaluation at the point $y$. We will use the fact that $\Phi_{y}$ is $\varepsilon$-disjointness preserving if $\Phi$ is $\varepsilon$-disjointness preserving to obtain the following extension of Theorem[1.

Corollary. Let $X$ and $Y$ be compact Hausdorff spaces and $\varepsilon \geq 0$. Then for every continuous $\varepsilon$-disjointness preserving linear mapping $\Phi: \mathcal{C}(X) \rightarrow \mathcal{C}(Y)$ there exists a continuous disjointness preserving linear mapping $\Psi: \mathcal{C}(X) \rightarrow \mathcal{C}(Y)$ satisfying

$$
\|\Phi(f)-\Psi(f)\| \leq 20 \sqrt{\varepsilon}\|f\| \quad \text { for all } f \in \mathcal{C}(X)
$$

For unbounded $\varepsilon$-disjointness preserving linear functionals we have a superstability phenomenon.

Theorem 2. Let $X$ be a compact Hausdorff space and $\Phi$ an unbounded linear functional on $\mathcal{C}(X)$. If there exists an $\varepsilon \geq 0$ such that $\Phi$ is $\varepsilon$-disjointness preserving, then $\Phi$ is disjointness preserving.

Having this result, it is tempting to conjecture that the same superstability phenomenon occurs also for unbounded $\varepsilon$-disjointness preserving mappings between $\mathcal{C}(X)$ and $\mathcal{C}(Y)$. The following example shows that this is not the case. So, we face a completely different situation compared to Theorem 1 and its Corollary.

Example. Let $X=\beta \mathbb{N}$ be the Stone-Čech compactification of the set of all positive integers and let $Y=[0,1]$ with the usual topology. Further, let $G$ be the linear functional on $\mathcal{C}(X)$, defined by $G(f)=f(1)+f(2)$, and $F$ any unbounded disjointness preserving linear functional on $\mathcal{C}(X)$ (the existence of such functionals will be proved in Lemma 3 in the next section). Then the linear mapping $\Phi: \mathcal{C}(X) \rightarrow \mathcal{C}(Y)$ given by

$$
\Phi(f)(y)= \begin{cases}\sqrt{\varepsilon}(1-2 y) G(f) & \text { if } \quad 0 \leq y \leq \frac{1}{2} \\ (2 y-1) F(f) & \text { if } \quad \frac{1}{2}<y \leq 1\end{cases}
$$

is unbounded and $\varepsilon$-disjointness preserving (when checking this property one has to use the fact that $|G(f) G(g)| \leq\|f\|\|g\|$ whenever $f g=0$ ) but it is not disjointness preserving.

In particular, it follows from Theorem 1 and Theorem 2 that the $\varepsilon$-disjointness property is stable for all linear functionals on $\mathcal{C}(X)$. Is this true also for linear mappings between $\mathcal{C}(X)$ and $\mathcal{C}(Y)$ ? More precisely, can we omit the continuity assumption in the Corollary? Our last theorem gives an affirmative answer under the additional assumption of surjectivity, which is automatically satisfied in the case of nonzero functionals.

Theorem 3. Let $X$ and $Y$ be compact Hausdorff spaces and $\varepsilon \geq 0$. Then for every surjective $\varepsilon$-disjointness preserving linear mapping $\Phi: \mathcal{C}(X) \rightarrow \mathcal{C}(Y)$ there exists a disjointness preserving linear mapping $\Psi: \mathcal{C}(X) \rightarrow \mathcal{C}(Y)$ satisfying

$$
\|\Phi(f)-\Psi(f)\| \leq 20 \sqrt{\varepsilon}\|f\| \quad \text { for all } f \in \mathcal{C}(X) .
$$

\section{Proofs}

In order to prove Theorem 1 we will need the following lemma. For the sake of completeness we will present its proof which goes through in the same way as the proof of Theorem 4.1 in [4]. 
Lemma 1. Let $X$ be a compact Hausdorff space and $\varepsilon \geq 0$. If $\Phi$ is a positive continuous $\varepsilon$-disjointness preserving linear functional on $\mathcal{C}(X)$ satisfying $\|\Phi\|>$ $3 \sqrt{\varepsilon}$, and if $\lambda$ is a positive measure which represents $\Phi$, then there exists a unique $x_{0} \in X$ such that $\lambda\left(\left\{x_{0}\right\}\right) \geq\|\Phi\|-\frac{2 \sqrt{\varepsilon}}{3}$ and

$$
\|\| \Phi\left\|\delta_{x_{0}}-\Phi\right\| \leq \frac{4}{3} \sqrt{\varepsilon}
$$

Proof of Lemma[1. Let us denote

$$
\Phi_{1}=\Phi /\|\Phi\|
$$

Since $\Phi_{1}$ is a positive linear functional, there exists by the Riesz theorem a unique positive regular measure $\mu$ such that

$$
\Phi_{1}(f)=\int_{X} f d \mu \quad \text { for all } f \in \mathcal{C}(X),
$$

and $\mu(X)=\Phi_{1}\left(1_{X}\right)=\left\|\Phi_{1}\right\|=1$. So $\mu$ is a probability measure. Let

$$
\alpha=\sup \{\mu(\{x\}): x \in X\} .
$$

First, we will show that $\alpha \geq \frac{1}{3}$. Suppose, contrary to our claim, that $\alpha<\frac{1}{3}$. Since $\mu$ is a regular measure, there exists for every $x \in X$ an open set $N_{x}$ containing $x$ such that $\mu\left(N_{x}\right)<\frac{1}{3}$. The family $\left\{N_{x}: x \in X\right\}$ is a covering of the compact space $X$, so there exists a finite subcovering $\left\{N_{1}, \ldots, N_{k}\right\}$. Let us define $G_{0}=\emptyset, G_{i}=\bigcup_{j=1}^{i} N_{j}$, $i=1, \ldots, k$. Then $\emptyset=G_{0} \subset G_{1} \subset \ldots \subset G_{k}=X$ and $\mu\left(G_{i}\right)-\mu\left(G_{i-1}\right)=$ $\mu\left(G_{i} \backslash G_{i-1}\right) \leq \mu\left(N_{i}\right)<\frac{1}{3}$, so there exists an $i_{0}$ such that $\frac{1}{3}<\mu\left(G_{i_{0}}\right)<\frac{2}{3}$. Because the measure $\mu$ is regular, there exists a compact set $F_{1} \subset G_{i_{0}}$ with $\mu\left(F_{1}\right)>\frac{1}{3}$. The compact set $F_{2}=G_{i_{0}}^{C}$ also has the property that $\mu\left(F_{2}\right)=\mu(X)-\mu\left(G_{i_{0}}\right)>\frac{1}{3}$. Since $F_{1}$ and $F_{2}$ are disjoint closed sets and $X$ is a compact Hausdorff space, there exist disjoint open sets $U_{1} \supset F_{1}, U_{2} \supset F_{2}$ and, by Urysohn's lemma, functions $h_{1}, h_{2} \in \mathcal{C}(X)$ such that $0 \leq h_{i} \leq 1_{X},\left.h_{i}\right|_{U_{i}^{C}} \equiv 0$ and $\left.h_{i}\right|_{F_{i}} \equiv 1, i=1,2$, so $h_{1} h_{2}=0$. It follows on one side that

$$
\Phi_{1}\left(h_{1}\right) \Phi_{1}\left(h_{2}\right) \leq \frac{\varepsilon}{\|\Phi\|^{2}}\left\|h_{1}\right\|\left\|h_{2}\right\|<\frac{1}{9},
$$

since $\|\Phi\|>3 \sqrt{\varepsilon}$, but on the other side

$$
\Phi_{1}\left(h_{1}\right) \Phi_{1}\left(h_{2}\right) \geq \mu\left(F_{1}\right) \mu\left(F_{2}\right)>\frac{1}{9},
$$

which is a contradiction. So $\alpha \geq \frac{1}{3}$.

There exists an $x_{0} \in X$ such that $\mu\left(\left\{x_{0}\right\}\right)=\alpha$. If not, there would be infinitely many $x \in X$ with $\mu(\{x\})>c$ for any constant $0<c<\frac{1}{3}$, which is not possible since $\mu(X)=1$.

Let $\alpha<\alpha^{\prime}<1$. Then, because of the regularity of the measure $\mu$, there exists an open neighborhood $N_{x_{0}}$ of $x_{0}$ satisfying $\mu\left(N_{x_{0}}\right)<\alpha^{\prime}$. The space $X$ is compact Hausdorff, so there exist disjoint open sets $V_{1} \supset\left\{x_{0}\right\}, V_{2} \supset N_{x_{0}}^{C}$ and, by Urysohn's lemma, functions $h_{1}, h_{2} \in \mathcal{C}(X)$ such that $0 \leq h_{i} \leq 1_{X},\left.h_{i}\right|_{V_{i}^{C}} \equiv 0, i=1,2$, and $h_{1}\left(x_{0}\right)=1,\left.h_{2}\right|_{N_{x_{0}}^{C}} \equiv 1$, so $h_{1} h_{2}=0$. Then

$$
\alpha\left(1-\alpha^{\prime}\right)<\mu\left(\left\{x_{0}\right\}\right) \mu\left(N_{x_{0}}^{C}\right) \leq \Phi_{1}\left(h_{1}\right) \Phi_{1}\left(h_{2}\right) \leq \frac{\varepsilon}{\|\Phi\|^{2}}\left\|h_{1}\right\|\left\|h_{2}\right\|
$$


for all $\alpha^{\prime}>\alpha$, and hence $\alpha(1-\alpha) \leq \frac{\varepsilon}{\|\Phi\|^{2}}$. Let $\delta=\frac{\varepsilon}{\|\Phi\|^{2}}$. Because

$$
\frac{1}{9}>\delta \geq \alpha(1-\alpha)=\frac{1}{4}-\left(\alpha-\frac{1}{2}\right)^{2}
$$

and $\alpha \geq \frac{1}{3}$, it follows that actually $\alpha>\frac{1}{2}+\frac{\sqrt{5}}{6}$, so

$$
\alpha \geq \frac{1}{2}+\sqrt{\frac{1}{4}-\delta} .
$$

And since $\frac{1}{4}-\delta \geq\left(\frac{1}{2}-2 \delta\right)^{2}$, we obtain that

$$
\alpha \geq 1-2 \delta .
$$

Let $f \in \mathcal{C}(X)$ be such that $\|f\| \leq 1$. Then

$$
\begin{aligned}
\left|\delta_{x_{0}}(f)-\Phi_{1}(f)\right| & =\left|\int_{X} f\left(x_{0}\right) d \mu-\int_{X} f d \mu\right|=\left|\int_{X \backslash\left\{x_{0}\right\}}\left(f\left(x_{0}\right)-f\right) d \mu\right| \\
& \leq 2(1-\alpha) \leq 4 \delta
\end{aligned}
$$

and

$$
\|\| \Phi\left\|\delta_{x_{0}}-\Phi\right\| \leq 4 \frac{\varepsilon}{\|\Phi\|} \leq \frac{4}{3} \sqrt{\varepsilon} .
$$

If $\alpha=1$, then $\delta_{x_{0}}=\Phi_{1}$, so $\|\Phi\| \delta_{x_{0}}=\Phi$ and (11) is true also in this case.

Since $\alpha>\frac{1}{2}$ and $\mu$ is a probability measure, there is only one $x_{0} \in X$ such that $\mu\left(\left\{x_{0}\right\}\right)=\alpha$. Also, because $\lambda=\|\Phi\| \mu$, we have $\lambda\left(\left\{x_{0}\right\}\right) \geq\|\Phi\|-2\|\Phi\| \delta \geq$ $\|\Phi\|-\frac{2 \sqrt{\varepsilon}}{3}$.

Proof of Theorem [1. Let $\Phi$ be a nontrivial bounded functional. Then there exists a positive linear functional $|\Phi|$ on $\mathcal{C}(X)$ called the modulus of $\Phi$ and defined by

$$
|\Phi|(f)=\sup \{|\Phi(u)|:|u| \leq f\}
$$

for every nonnegative real $f \in \mathcal{C}(X)$ (see the proof of Theorem 6.19 in [6] and also 7]). By the Riesz theorem there exists a unique measure $\mu$ such that

$$
\Phi(f)=\int_{X} f d \mu \quad \text { for all } f \in \mathcal{C}(X),
$$

and, by the argument given in the proof of Theorem 6.19 in [6], it follows that also

$$
|\Phi|(f)=\int_{X} f \bar{w} d \mu=\int_{X} f d|\mu| \quad \text { for all } f \in \mathcal{C}(X),
$$

where $w \in \mathcal{L}^{1}(|\mu|)$ with $|w|=1$ a.e. $[|\mu|]$.

Let us prove that $|\Phi|$ is an $\varepsilon$-disjointness preserving functional if $\Phi$ is $\varepsilon$-disjointness preserving. If $f, g \geq 0$ and $f g=0$, then note that for any $u, v \in \mathcal{C}(X)$ satisfying $|u| \leq f,|v| \leq g$ also $u v=0$, and so

$$
\begin{aligned}
|\Phi|(f)|\Phi|(g) & =\sup \{|\Phi(u)|:|u| \leq f\} \sup \{|\Phi(v)|:|v| \leq g\} \\
& =\sup \{|\Phi(u) \Phi(v)|:|u| \leq f,|v| \leq g\} \leq \varepsilon\|f\|\|g\| .
\end{aligned}
$$

Since

$$
|| \Phi|(f)|=\left|\int_{X} f d\right| \mu|| \leq \int_{X}|f| d|\mu|=|\Phi|(|f|)
$$


for every $f \in \mathcal{C}(X)$, it follows that

$$
|| \Phi|(f)| \Phi|(g)| \leq|\Phi|(|f|)|\Phi|(|g|) \leq \varepsilon\|f\|\||| g\|
$$

if $f$ and $g$ are continuous functions with $f g=0$.

Since $|\Phi|$ is a positive linear functional, we have

$$
\||\Phi|\|=|\Phi|\left(1_{X}\right)=\sup \left\{|\Phi(u)|:|u| \leq 1_{X}\right\}=\|\Phi\| .
$$

If $\|\Phi\| \leq 3 \sqrt{\varepsilon}$, then $\Phi$ is near the zero functional.

It remains to consider the case $\|\Phi\|>3 \sqrt{\varepsilon}$. This will be done in the following lemma, which will also be used later on.

Lemma 2. Let $\Phi$ be a continuous $\varepsilon$-disjointness preserving linear functional on $\mathcal{C}(X)$ and suppose $\|\Phi\|>3 \sqrt{\varepsilon}$. Then there exists a unique $x_{0} \in X$ such that

$$
\left\|\Phi-w\left(x_{0}\right)\right\| \Phi\left\|\delta_{x_{0}}\right\| \leq 3 \sqrt{\varepsilon},
$$

where $\left|w\left(x_{0}\right)\right|=1$.

Proof of Lemma国. Since $|\Phi|$ is a positive continuous $\varepsilon$-disjointness preserving linear functional, it follows by Lemma 1 and (4) that there exists a unique $x_{0} \in X$ such that

$$
\|\| \Phi\left\|\delta_{x_{0}}-|\Phi|\right\| \leq \frac{4}{3} \sqrt{\varepsilon} .
$$

Let us prove that $w\left(x_{0}\right)\|\Phi\| \delta_{x_{0}}$ is near the linear functional $\Phi$. It follows by (2), (3), (6) and Lemma 1 that

$$
\begin{aligned}
\left|\Phi(f)-w\left(x_{0}\right)\|\Phi\| f\left(x_{0}\right)\right| \leq & \left|\Phi(f)-w\left(x_{0}\right)\right| \Phi|(f)| \\
& +\left|w\left(x_{0}\right)\right| \Phi\left|(f)-w\left(x_{0}\right)\|\Phi\| f\left(x_{0}\right)\right| \\
\leq & \left|\int_{X} f\left(w-w\left(x_{0}\right)\right) d\right| \mu||+\frac{4}{3} \sqrt{\varepsilon} \\
\leq & 2\|f\| \int_{X \backslash\left\{x_{0}\right\}} d|\mu|+\frac{4}{3} \sqrt{\varepsilon} \\
\leq & \frac{8}{3} \sqrt{\varepsilon}
\end{aligned}
$$

for $f \in \mathcal{C}(X)$ satisfying $\|f\| \leq 1$, and so the proof is completed.

Proof of the Corollary. Let $\varepsilon>0$ and let $\Phi$ be a continuous $\varepsilon$-disjointness preserving linear mapping from $\mathcal{C}(X)$ to $\mathcal{C}(Y)$. Clearly, for every $y \in Y$ the linear functional $\Phi_{y}$ is $\varepsilon$-disjointness preserving. For any $K \geq 0$ we define the set $Y_{K}=\left\{y \in Y:\left\|\Phi_{y}\right\|>K\right\}$. Take any $y \in Y_{K}$. There exists a function $f \in \mathcal{C}(X),\|f\| \leq 1$, satisfying $|\Phi(f)(y)|=\left|\Phi_{y}(f)\right|>K$ and, since $\Phi(f) \in \mathcal{C}(Y)$, a neighborhood $U$ of $y$ such that $|\Phi(f)(z)|>K$ for all $z \in U$. It follows that $\left\|\Phi_{z}\right\| \geq|\Phi(f)(z)|>K$ for all $z \in U$, so $U \subset Y_{K}$ and the set $Y_{K}$ is open. By Lemma 2 there exists for any continuous $\varepsilon$-disjointness preserving linear functional $\Upsilon$ on $\mathcal{C}(X)$ with $\|\Upsilon\|>3 \sqrt{\varepsilon}$ a unique $x_{\Upsilon} \in X$ satisfying condition (5). Define a mapping $\phi: Y_{6 \sqrt{\varepsilon}} \rightarrow X$ by

$$
\phi(y)=x_{\Phi_{y}} .
$$

Let us show that $\phi$ is continuous. Assume, on the contrary, that there exist a $y \in Y_{6 \sqrt{\varepsilon}}$ and an open neighborhood $V$ of $\phi(y)$ such that every neighborhood $U$ of $y$ contains a point $z \in Y_{6 \sqrt{\varepsilon}}$ with $\phi(z) \notin V$. Since $X$ is a compact Hausdorff 
space, there exists a function $f \in \mathcal{C}(X)$ satisfying supp $f \subset V, 0 \leq f \leq 1_{X}$ and $f(\phi(y))=1$. Then by Lemma 2

$$
\left|\Phi_{y}(f)-w_{y}(\phi(y))\left\|\Phi_{y}\right\| f(\phi(y))\right|=\left|\Phi(f)(y)-w_{y}(\phi(y))\left\|\Phi_{y}\right\|\right| \leq 3 \sqrt{\varepsilon} .
$$

Because $\left|w_{y}(\phi(y))\right|=1$ and $\left\|\Phi_{y}\right\|>6 \sqrt{\varepsilon}$,

$$
|\Phi(f)(y)| \geq\left|w_{y}(\phi(y))\right|\left\|\Phi_{y}\right\|-3 \sqrt{\varepsilon}>3 \sqrt{\varepsilon} .
$$

Let $U \subset Y_{6 \sqrt{\varepsilon}}$ be a neighborhood of $y$ and let $z \in U$ be such that $\phi(z) \notin V$. Then by Lemma 2

$$
|\Phi(f)(z)|=\left|\Phi(f)(z)-w_{z}(\phi(z))\left\|\Phi_{z}\right\| f(\phi(z))\right| \leq 3 \sqrt{\varepsilon},
$$

which is a contradiction since $\Phi(f) \in \mathcal{C}(Y)$. So, $\phi$ is continuous.

Our next claim is that the closure of the open set $Y_{10 \sqrt{\varepsilon}}$ is a subset of the open set $Y_{6 \sqrt{\varepsilon}}$, that is,

$$
\overline{Y_{10 \sqrt{\varepsilon}}} \subset Y_{6 \sqrt{\varepsilon}} .
$$

Suppose, contrary to our claim, that there is a $y \in \overline{Y_{10 \sqrt{\varepsilon}}}$ such that $\left\|\Phi_{y}\right\| \leq 6 \sqrt{\varepsilon}$. Then there exists a neighborhood $U$ of $y$ such that

$$
\left|\Phi\left(1_{X}\right)(z)\right| \leq 7 \sqrt{\varepsilon} \quad \text { for all } z \in U .
$$

Because $y \in \overline{Y_{10 \sqrt{\varepsilon}}}$, there exists a $z_{0} \in Y_{10 \sqrt{\varepsilon}} \cap U$, so by Lemma 2

$$
\left|\Phi_{z_{0}}\left(1_{X}\right)\right| \geq\left\|\Phi_{z_{0}}\right\|-3 \sqrt{\varepsilon}>7 \sqrt{\varepsilon}
$$

which is impossible.

It follows that ${\overline{Y_{10 \sqrt{\varepsilon}}}}^{C} \cup Y_{6 \sqrt{\varepsilon}}=Y$. The space $Y$ is compact Hausdorff, so there exists a function $h \in \mathcal{C}(Y)$ satisfying $\operatorname{supp} h \subset Y_{6 \sqrt{\varepsilon}}, 0 \leq h \leq 1_{Y}$ and $h(y)=1$ for all $y \in \overline{Y_{10 \sqrt{\varepsilon}}}$. Pick any $x \in X$ and extend the mapping (7) to $\phi: Y \rightarrow X$ by $\phi(y)=x$ for all $y \in Y \backslash Y_{6 \sqrt{\varepsilon}}$. Let us define a linear mapping $\Psi$ on $\mathcal{C}(X)$ by

$$
\Psi(f)(y)=h(y) \Phi\left(1_{X}\right)(y) f(\phi(y)), \quad f \in \mathcal{C}(X), y \in Y .
$$

Since supp $h \subset Y_{6 \sqrt{\varepsilon}}$ and the mappings $h, \Phi\left(1_{X}\right), \phi$ are continuous on $Y_{6 \sqrt{\varepsilon}}, \Psi$ maps from $\mathcal{C}(X)$ to $\mathcal{C}(Y)$. The mapping (9) is obviously linear and disjointness preserving and it is also continuous because for any $f \in \mathcal{C}(X)$

$$
\|\Psi(f)\|=\sup _{y \in Y}\left|h(y) \Phi\left(1_{X}\right)(y) f(\phi(y))\right| \leq\|\Phi\|\|f\| .
$$

It remains to prove that $\Psi$ is near $\Phi$. In order to do that take $f \in \mathcal{C}(X)$ with $\|f\| \leq 1$. If $y \in Y \backslash Y_{10 \sqrt{\varepsilon}}$, then

$$
|\Phi(f)(y)-\Psi(f)(y)| \leq\left|\Phi_{y}(f)\right|+\left|\Phi_{y}\left(1_{X}\right)\right| \leq 20 \sqrt{\varepsilon} .
$$

And if $y \in Y_{10 \sqrt{\varepsilon}}$, then by Lemma 2

$$
\begin{aligned}
|\Phi(f)(y)-\Psi(f)(y)| \leq & \left|\Phi_{y}(f)-w_{y}(\phi(y))\left\|\Phi_{y}\right\| f(\phi(y))\right| \\
& +\left|w_{y}(\phi(y))\left\|\Phi_{y}\right\| f(\phi(y))-h(y) \Phi_{y}\left(1_{X}\right) f(\phi(y))\right| \\
\leq & 3 \sqrt{\varepsilon}+\left|w_{y}(\phi(y))\left\|\Phi_{y}\right\|-\Phi_{y}\left(1_{X}\right)\right||f(\phi(y))| \\
\leq & 3 \sqrt{\varepsilon}+\left|w_{y}(\phi(y))\left\|\Phi_{y}\right\| 1_{X}(\phi(y))-\Phi_{y}\left(1_{X}\right)\right| \\
\leq & 6 \sqrt{\varepsilon} .
\end{aligned}
$$

So,

$$
\|\Phi-\Psi\| \leq 20 \sqrt{\varepsilon} .
$$


Proof of Theorem 圆 Let $\varepsilon>0$ and let $\Phi$ be $\varepsilon$-disjointness preserving. First, we will show that $\Phi(f) \Phi(g)=0$ whenever supp $f \cap \operatorname{supp} g=\emptyset$. Suppose this is false. Then we can find $f, g \in \mathcal{C}(X)$ satisfying $\|f\|,\|g\| \leq 1$ and $\operatorname{supp} f \cap \operatorname{supp} g=\emptyset$, for which $\Phi(f) \neq 0$ and $\Phi(g) \neq 0$. If we define $U_{1}=(\operatorname{supp} g)^{C}$ and $U_{2}=(\operatorname{supp} f)^{C}$, then $U_{1} \cup U_{2}=X$. So, since $X$ is a compact Hausdorff space, there exist $h_{1}, h_{2} \in \mathcal{C}(X)$ such that $0 \leq h_{i} \leq 1_{X}, \operatorname{supp} h_{i} \subset U_{i}, i=1,2$, and $h_{1}+h_{2}=1_{X}$. Because $\Phi$ is an unbounded functional, there exists a function $k \in \mathcal{C}(X)$ with $\|k\| \leq 1$ and

$$
|\Phi(k)|>\frac{\varepsilon}{|\Phi(f)|}+\frac{\varepsilon}{|\Phi(g)|} .
$$

Since $f k h_{2}=0$, we have

$$
\left|\Phi\left(k h_{2}\right)\right| \leq \frac{\varepsilon}{|\Phi(f)|}
$$

so

$$
\left|\Phi\left(k h_{1}\right)\right| \geq|\Phi(k)|-\frac{\varepsilon}{|\Phi(f)|}>\frac{\varepsilon}{|\Phi(g)|} .
$$

Also, because $g k h_{1}=0$, it follows that

$$
\left|\Phi\left(k h_{1}\right)\right| \leq \frac{\varepsilon}{|\Phi(g)|},
$$

a contradiction.

Further, suppose $|\Phi(f) \Phi(g)| \neq 0$, where $f, g \in \mathcal{C}(X)$ with $f g=0$. Then $F_{1}=$ $\left\{x:|f(x)| \geq \frac{|\Phi(f) \Phi(g)|}{\varepsilon\|g\|}\right\}$ and $F_{2}=\operatorname{supp} g$ are disjoint closed sets, so there exists an $h \in \mathcal{C}(X)$ such that $0 \leq h \leq 1_{X},\left.h\right|_{F_{1}} \equiv 1$ and $\operatorname{supp} h \subset(\operatorname{supp} g)^{C}$. Since $\operatorname{supp} h f \cap \operatorname{supp} g=\emptyset$, it follows that $\Phi(h f)=0$ and $|\Phi(f-h f)|=|\Phi(f)|$. We also know that $(f-h f) g=0$ and $\|f-h f\|<\frac{|\Phi(f) \Phi(g)|}{\varepsilon\|g\|}$, so

$$
|\Phi(f-h f) \Phi(g)|=|\Phi(f) \Phi(g)|>\varepsilon\|f-h f\|\|g\|,
$$

which contradicts the assumption that $\Phi$ is $\varepsilon$-disjointness preserving.

It should be mentioned that the idea of the proof of the following lemma is similar to the one used by Abramovich [1. Example 1] (see also Jarosz [2, Example]).

Lemma 3. There exists an unbounded disjointness preserving linear functional on $\mathcal{C}(X)$.

Proof of Lemma 3. Let $\mathbb{N}$ be the space of all positive integers with the discrete topology and let $\beta \mathbb{N}$ be the Stone-Cech compactification of $\mathbb{N}$. We will show that there exists an unbounded linear functional on $\mathcal{C}(\beta \mathbb{N})$ which is disjointness preserving. It is sufficient to define this functional on a Hamel basis. So, take an arbitrary element $x_{0}$ from $\beta \mathbb{N} \backslash \mathbb{N}$ and let $\left\{e_{\lambda}: \lambda \in \Lambda\right\}$ be a Hamel basis of the subspace of all functions $f \in \mathcal{C}(\beta \mathbb{N})$ having the property that $x_{0} \notin \operatorname{supp} f$. If the function $h$ is given by $h(n)=\frac{1}{n}, n \in \mathbb{N}$, and if $\beta h$ is its extension to the $\beta \mathbb{N}$, then $x_{0} \in \operatorname{supp} \beta h$, since $\mathbb{N}$ is a dense subset in $\beta \mathbb{N}$. It follows that $\{\beta h\} \cup\left\{e_{\lambda}: \lambda \in \Lambda\right\}$ is a linearly independent subset of $\mathcal{C}(\beta \mathbb{N})$, so it can be completed to the Hamel basis $\{\beta h\} \cup\left\{e_{\lambda}: \lambda \in \Lambda\right\} \cup\left\{f_{\pi}: \pi \in \Pi\right\}$ of the vector space $\mathcal{C}(\beta \mathbb{N})$. On this basis we define the linear functional $\Phi$ to be $\Phi(\beta h)=1, \Phi\left(e_{\lambda}\right)=0, \lambda \in \Lambda$, and $\Phi\left(f_{\pi}\right)=0$, $\pi \in \Pi$. 
Let us show that $\Phi$ is unbounded. For every positive integer $m$ we define a function $h_{m}$ on $\mathbb{N}$ by

$$
h_{m}(n)= \begin{cases}\frac{1}{n} & \text { if } n \leq m, \\ 0 & \text { if } n>m,\end{cases}
$$

and then extend it to a continuous function $\beta h_{m}$ on $\beta \mathbb{N}$. Because $(\beta \mathbb{N} \backslash \mathbb{N}) \cap$ $\operatorname{supp} \beta h_{m}=\emptyset$ for every positive integer $m$ and because $\beta h(x)=0$ if $x \in \beta \mathbb{N} \backslash \mathbb{N}$, the limit of the sequence $\left\{\beta h_{m}\right\}$ equals $\beta h$. The functional $\Phi$ is therefore unbounded since $\Phi\left(\beta h_{m}\right)=0$ for every positive integer $m$ and $\Phi(\beta h)=1$.

Let us prove that it is also disjointness preserving. Suppose $f g=0$, where $f, g \in \mathcal{C}(\beta \mathbb{N})$. Then $N_{1}=\{n \in \mathbb{N}: f(n) \neq 0\}, N_{2}=\{n \in \mathbb{N}: g(n) \neq 0\}$ and $N_{3}=\{n \in \mathbb{N}: f(n)=g(n)=0\}$ are pairwise disjoint sets and $N_{1} \cup N_{2} \cup N_{3}=\mathbb{N}$. Note that $f(x)=0$ for every $x \in \beta \mathbb{N} \backslash \overline{N_{1}}$ and $g(x)=0$ for every $x \in \beta \mathbb{N} \backslash \overline{N_{2}}$. We define a function $k$ on $\mathbb{N}$ by $k(n)=0$ if $n \in N_{1} \cup N_{3}$ and $k(n)=1$ if $n \in N_{2}$, and then extend it to a continuous function $\beta k$ on $\beta \mathbb{N}$. Since $\beta k^{-1}(0) \supset \overline{N_{1}}$ and $\beta k^{-1}(1) \supset \overline{N_{2}}$, at most one of the sets $\overline{N_{1}}$ or $\overline{N_{2}}$ contains $x_{0}$, so at least one of $\Phi(f)$ and $\Phi(g)$ equals zero, meaning that $\Phi$ is disjointness preserving.

We will need the following lemma in the proof of the last theorem.

Lemma 4. Suppose $X$ and $Y$ are compact Hausdorff spaces and $\Phi: \mathcal{C}(X) \rightarrow \mathcal{C}(Y)$ is a linear mapping. Then

$$
B=\left\{y \in Y: \Phi_{y} \text { is a bounded linear functional }\right\}
$$

and

$$
D=\left\{y \in Y: \Phi_{y} \text { is a disjointness preserving linear functional }\right\}
$$

are closed subsets of $Y$.

Proof of Lemma 㤢. Because $\left\{\Phi_{y}: y \in B\right\}$ is a collection of continuous linear functionals on the Banach space $\mathcal{C}(X)$ such that for every $f \in \mathcal{C}(X)$ the set $\left\{\left|\Phi_{y}(f)\right|: y \in B\right\}$ is bounded by $\|\Phi(f)\|$, it follows by the principle of uniform boundedness that there exists a constant $M$ such that $\left\|\Phi_{y}\right\| \leq M$ for every $y \in B$. Also, since

$$
\sup _{y \in \bar{B}}|\Phi(f)(y)|=\sup _{y \in B}|\Phi(f)(y)| \leq M\|f\|
$$

for every $f \in \mathcal{C}(X)$, the set $B$ is closed.

To deduce that $D$ is closed, take $f, g \in \mathcal{C}(X)$ satisfying $f g=0$. Then

$$
\sup _{y \in \bar{D}}|\Phi(f) \Phi(g)(y)|=\sup _{y \in D}|\Phi(f) \Phi(g)(y)|=0
$$

and $D$ is closed.

In the proof of Theorem 3 we will use a result of Jarosz [2 Theorem].

Proof of Theorem 3 . Let $\Phi$ be a surjective $\varepsilon$-disjointness preserving linear mapping from $\mathcal{C}(X)$ onto $\mathcal{C}(Y)$ and let $B$ and $D$ be the closed subsets of $Y$ defined in Lemma 4. Then $B^{C}$ is a subset of $D$ by Theorem 2. We will assume that $B^{C} \neq \emptyset$ and $D^{C} \neq \emptyset$ since these special cases follow easily from previous results. Let $\Phi_{B}: \mathcal{C}(X) \rightarrow \mathcal{C}(B)$ be given by

$$
\Phi_{B}(f)=\left.\Phi(f)\right|_{B}, \quad f \in \mathcal{C}(X) .
$$


Since $\left\{\left|\delta_{y} \circ \Phi_{B}(f)\right|: y \in B\right\}$ is bounded for every $f \in \mathcal{C}(X)$, it follows by the principle of uniform boundedness that $\Phi_{B}$ is bounded. It is clear that $\Phi_{B}$ is also $\varepsilon$-disjointness preserving, so we can define a continuous mapping $\phi_{B}: B_{6 \sqrt{\varepsilon}} \rightarrow X$ by (7), where $B_{K}=\left\{y \in B:\left\|\delta_{y} \circ \Phi_{B}\right\|>K\right\}$.

In a similar way we define $\Phi_{D}: \mathcal{C}(X) \rightarrow \mathcal{C}(D)$ by $\Phi_{D}(f)=\left.\Phi(f)\right|_{D}$, where $f \in$ $\mathcal{C}(X)$. Then $\Phi_{D}$ is a disjointness preserving linear mapping. So, by [2] Theorem], the set $D$ is a disjoint union of three sets $D_{1}, D_{2}$ and $D_{3}$, where $D_{2}=\{y \in D$ : $\delta_{y} \circ \Phi_{D}$ is unbounded $\}, D_{3}=\left\{y \in D: \delta_{y} \circ \Phi_{D}=0\right\}$ and $D_{1}=D \backslash\left(D_{2} \cup D_{3}\right)$, and there exists a continuous mapping $\phi_{D}: D_{1} \cup D_{2} \rightarrow X$ such that $\Phi_{D}(f)(y)=$ $\Phi_{D}\left(1_{X}\right)(y) f\left(\phi_{D}(y)\right)$ for every $y \in D_{1}, f \in \mathcal{C}(X)$, and $\phi_{D}\left(D_{2}\right)$ is a finite set $F=$ $\left\{x_{1}, \ldots, x_{n}\right\}$.

Because every function $f \in \mathcal{C}(D)$ can be extended to a function $f_{1} \in \mathcal{C}(Y)$ and because the mapping $\Phi$ is surjective, it follows that $\Phi_{D}$ is also surjective. So, the set $D_{3}$ is empty and $\phi_{D}$ is defined on the whole set $D$. Then $\phi_{D}^{-1}\left(x_{1}\right), \ldots, \phi_{D}^{-1}\left(x_{n}\right)$ are pairwise disjoint closed sets in $Y$ since $\phi_{D}$ is continuous and $D$ is closed in $Y$.

Let us show that $\phi_{D}(y)=\phi_{B}(y)$ for every $y \in B_{6 \sqrt{\varepsilon}} \cap D$. Otherwise there would exist a $y \in B_{6 \sqrt{\varepsilon}} \cap D$ and a $g \in \mathcal{C}(X)$ with $g\left(\phi_{D}(y)\right)=1$ and $g\left(\phi_{B}(y)\right)=0$. Then $\Phi\left(1_{X}\right)(y)=\Phi\left(1_{X}\right)(y) g\left(\phi_{D}(y)\right)=\Phi(g)(y)=w_{y}\left(\phi_{B}(y)\right)\left\|\Phi_{y}\right\| g\left(\phi_{B}(y)\right)=0$ by (5) and (7), which means that $y \in D_{3}$, a contradiction.

Next, we claim that $\phi_{D}$ is injective on $D_{1}$. Assume, on the contrary, that there exist different $y_{1}, y_{2} \in D_{1}$ such that $\phi_{D}\left(y_{1}\right)=\phi_{D}\left(y_{2}\right)$. But then any function $g \in \mathcal{C}(D)$, for which $g\left(y_{1}\right)=0$ and $g\left(y_{2}\right)=1$, would not be in the image of $\Phi_{D}$, a contradiction.

If we define $N_{i}=\phi_{D}^{-1}\left(x_{i}\right)$, then there is at most one element from $D_{1}$ in each of the closed sets $N_{i}, i=1, \ldots, n$. Let $\left\{N_{i}: i \in I \subset\{1, \ldots, n\}\right\}$ be the collection of sets for which there exists a $y_{i} \in N_{i}$ such that $\left\|\Phi_{y_{i}}\right\| \leq 6 \sqrt{\varepsilon}$. Let us in addition denote $N_{0}=\overline{B_{10 \sqrt{\varepsilon}}} \cup\left(\bigcup_{i \in\{1, \ldots, n\} \backslash I} N_{i}\right)$ and $I_{0}=\{0\} \cup I$. By (으), $\left\{N_{i}: i \in I_{0}\right\}$ is a family of pairwise disjoint closed subsets of the compact Hausdorff space $Y$, so there exist pairwise disjoint open sets $V_{0} \supset N_{0}$ and $U_{i} \supset N_{i}, i \in I$. Since $N_{0}$ is a subset of the open set $\left(B \backslash B_{6 \sqrt{\varepsilon}}\right)^{C}$, also $U_{0}=V_{0} \cap\left(B \backslash B_{6 \sqrt{\varepsilon}}\right)^{C}$ is an open set containing $N_{0}$. By Urysohn's lemma there exist $h_{i} \in \mathcal{C}(Y)$ such that $0 \leq h_{i} \leq 1_{Y}$, $\operatorname{supp} h_{i} \subset U_{i}$ and $h_{i}(y)=1$ for $y \in N_{i}, i \in I_{0}$. Define a mapping $\phi: Y \rightarrow X$ by

$$
\phi(y)= \begin{cases}\phi_{B}(y) & \text { if } y \in U_{0} \cap B, \\ x_{i} & \text { if } y \in U_{i}, i \in I, \\ x_{1} & \text { otherwise, }\end{cases}
$$

and a linear mapping $\Psi$ by

$$
\Psi(f)(y)= \begin{cases}\Phi(f)(y) & \text { if } y \in D_{2}, \\ \Phi\left(1_{X}\right)(y) f(\phi(y))\left(\sum_{i \in I_{0}} h_{i}(y)\right) & \text { if } y \in B,\end{cases}
$$

for every $f \in \mathcal{C}(X)$. Because $\phi$ is continuous on $B \cap\left(\bigcup_{i \in I_{0}} U_{i}\right)$ and $\operatorname{supp} \sum_{i \in I_{0}} h_{i} \subset$ $\bigcup_{i \in I_{0}} U_{i}$, we see that $\Psi(f)$ is continuous on $B$. And because $\phi_{D}(y)=\phi(y)$ and $\sum_{i \in I_{0}} h_{i}(y)=1$ for every $y \in \overline{D_{2}} \cap B$, it follows that $\Phi(f)(y)=\Psi(f)(y), y \in \overline{D_{2}} \cap B$. So, $\Psi(f) \in \mathcal{C}(Y)$ for every $f \in \mathcal{C}(X)$. Since $\Psi_{y}$ is a disjointness preserving functional for every $y \in B$ and also for every $y \in D_{2}, \Psi$ is also a disjointness preserving map. Finally, by (9), (10) and (11)

$$
\|\Phi(f)-\Psi(f)\| \leq 20 \sqrt{\varepsilon}\|f\| \quad \text { for all } f \in \mathcal{C}(X) .
$$




\section{REFERENCES}

[1] Y. A. Abramovich, Multiplicative representation of disjointness preserving operators, Nederl. Akad. Wetensch. Indag. Math. 45 (1983), 265-279. MR 85f:47040

[2] K. Jarosz, Automatic continuity of separating linear isomorphisms, Canad. Math. Bull. (2) 33 (1990), 139-144. MR 92j:46049

[3] K. Jarosz, Perturbations of Banach algebras, Lecture Notes in Mathematics 1120, Springer, Berlin, 1985. MR 86k:46074

[4] B. E. Johnson, Approximately multiplicative functionals, J. London Math. Soc. (2) 34 (1986), 489-510. MR 87k:46105

[5] B. E. Johnson, Approximately multiplicative maps between Banach algebras, J. London Math. Soc. (2) 37 (1988), 294-316. MR 89h:46072

[6] W. Rudin, Real and complex analysis, McGraw-Hill, New York, 1966. MR 35:1420

[7] H. H. Schaefer, Banach lattices and positive operators, Springer, Berlin, 1974. MR 54:11023

Faculty of Electrical Engineering, University of Luubluana, Slovenia

E-mail address: gregor.dolinar@fe.uni-lj.si 